\title{
A PERMEABLE REACTIVE BARRIER (PRB) FOR THE REMOVAL AND IMMOBILZATION OF SELENIUM IN SEEP WATER AND SHALLOW GROUNDWATER AT A PHOSPHATE MINE IN SOUTHERN IDAHO: RESULTS OF BENCH SCALE TESTING ${ }^{1}$
}

\author{
William J Walker ${ }^{2}$, David Tooke, Matthew Wright, Jeffrey Hamilton ${ }^{2}$, Cindy Schreier ${ }^{3}$ and \\ Jonathon Peterson
}

\begin{abstract}
A bench study was designed to determine the efficacy of a permeable reactive barrier (PRB) for removing elevated Se in groundwater and seep water at the toe of overburden storage area at a phosphate mine in Idaho. The bench testing was considered a first step in the pre-design considerations for developing an engineering and geochemical strategy for long-term water treatment options at the site.

The study consisted of three main parts: (1) characterization work designed to determine the basic chemistry of the site-water under consideration for treatment and the components of the proposed PRB. This data is a requirement for establishing the initial chemical conditions for assessing the efficacy of the PRB and its components. (2) batch leaching studies designed to assess the chemistry changes that each media component is expected to contribute to the overall water chemistry of the seep or groundwater in contact with the media. This batch study also allows a determination, prior to more extensive column testing, of whether unwanted chemicals or chemical changes will occur due to contract with the PRB media. (3) column studies consisting of vertical, 2-inch columns filled with the PRB media. Seep water and groundwater were delivered to the columns in an up-flow manner under a specified flow rate to allow sufficient HRT (12 and $24 \mathrm{hr}$ ) for Se reduction to occur.

The results of the studies indicated that the media proposed as components of the PRB posed no chemical changes of concern and resulted in rapid development of reducing conditions sufficient for Se reduction and immobilization. Column testing results were also very positive. The Se concentrations in seep water were initially about $10 \mathrm{mg} / \mathrm{L}$ and were reduced to about $0.2 \mathrm{mg} / \mathrm{L}$ after 25 pore volumes $(\mathrm{PV})$ and $0.1 \mathrm{mg} / \mathrm{L}$ after $50 \mathrm{PV}$. Increasing the hydraulic residence time (HRT) to $24 \mathrm{hr}$ (a factor of 2) decreased the Se to just above the 0.05 $\mathrm{mg} / \mathrm{L}$ water quality goal. The groundwater Se, initially about $1 \mathrm{mg} / \mathrm{L}$ was reduced to less than 0.02 in the first 3 hours of column contact time, well below the $0.05 \mathrm{mg} / \mathrm{L}$ water quality goal.

Overall, the results indicated that the PRB composition used in the study will be an effective means of meeting water quality goals at the site. The study provided much needed information on water and media composition information and the residence times required to accomplish Se reduction. Aside from Se reduction, no significant deleterious changes to water quality were observed when compared to primary water quality standards.
\end{abstract}

Additional Key Words: column and batch leaching, selenium reduction, selenium speciation, seep water, overburden, microbial reduction.

\footnotetext{
${ }^{1}$ This paper was submitted for consideration in JASMR as a Case Study and was not presented at one of the ASMR annual conferences.

${ }^{2}$ William J Walker, PhD. Senior Geochemist; David Tooke, PhD. Associate Geochemist. Matthew Wright, PE Senior Engineer, and Jonathon Peterson, Project Geochemist, NewFields Companies, Mining and Energy Services, Missoula, MT. Jeffrey Hamilton. Senior Environmental Engineering Manager. J.R. Simplot Corporation, Pocatello, ID. ${ }^{3}$ Cindy G. Schreier, PhD Chief Scientist, Prima Environmental, Sacramento CA.
}

DOI: http://dx.doi.org/10.21000/JASMR18030020 


\section{Introduction}

Elevated selenium (Se) in mine waters and mine wastes, primarily associated with phosphate rock mining, was identified in southeast Idaho in 1997 (Buck and Jones, 2003). The source of the problem was traced to overburden from the interbedded shales and mudstones of the Phosphoria formation (Desborough et al., 1999 and Herring et al., 1999) in the same region. Since that time, a consortium of Federal and State inter-agency groups along with mining companies' funded studies have sought to define the extent of the problem and to develop strategies to mitigate the impact of Se rich mine materials on local soil and water resources. The purpose of this work was to design and conduct a bench study to determine the efficacy of using a permeable reactive barrier (PRB) for removing elevated Se in groundwater and seep water at the toe of overburden storage area at a phosphate mine in Idaho (JR Simplot Conda/Woodall Mountain mine). The bench testing was considered a first step in the pre-design considerations for developing an engineering and geochemical strategy for long-term water treatment options at the site. The use of permeable reactive barriers (PRB) for contaminant removal in groundwater has been well studied (Blows et. al., 2000) and is a common means of passively and cost -effectively treating groundwater without the associated problems of extracting then treating and discharging or re-injecting the treated water. The PRBs can be composed of inorganic or organic media and mixtures of these to effect the desired change in subsurface geochemistry required for contaminant removal. In this study, the PRB was designed to create reducing conditions via organic matter decomposition and reduce selenate/selenite $\mathrm{S}$ to elemental Se presumably through microbially mediated microorganisms (Xia et al., 2013). As such, the PRB was constructed largely of organic substrates rather than the typical iron based (zero valent iron and/or iron oxyhydroxides) PRBs used in other Se removal processes (USEPA, 1998 and Blowes et al., 2000).

\section{Methods And Materials}

\section{Characterization of Water and Media Components}

Prior to the batch and column studies, the seep, groundwater, and media components were sampled and analyzed to determine the chemical composition of each. This exercise was conducted to set the initial conditions of water and media used in the batch and column studies. Approximately $200 \mathrm{~L}$ each of seep water and ground water were collected by Simplot and shipped to Prima Environmental in Sacramento, CA. Simplot collected water quality samples for laboratory analysis from the containers prior to shipment and then Prima Environmental re- 
sampled them twice after receipt and once before use in the batch and column studies to ensure that initial conditions were understood. At approximately the same time, samples of the media to be used in the study were collected and shipped to Prima, who in turn homogenized and subsampled the material and submitted each for chemical analysis.

The large plastic water containers were packed with ice for shipment to the laboratory. No preservatives were added to the water. Headspace was eliminated prior to sealing the containers to prevent additional oxygen uptake. While the water composition may have changed during shipment and storage, the overall composition in terms of major cations and anions (sample matrix) was expected to be minimally affected. Changes in Se concentrations, on the other hand, have been noted previously and would be countered by spiking the test waters with Se if necessary. This approach certainly fulfills the first step in the pre-design of a water treatment system, a step that seeks to answer the basic question "can the proposed PRB effectively remove Se from solution?" Once the bench testing is proven, a field pilot system will be constructed, and control over the influent water composition will be greatly improved. In addition, the final design is likely to include a collection basin/pond that will act as an equalization area for the influent to the PRB, so storage of the water at the mine will be similar to that used in the bench testing.

\section{Batch Leach Study}

The batch leach tests were designed to provide preliminary information for the column studies. For this work, the batch studies were conducted as described by ASTM Method D-4646-02, "24h Batch-Type Measurement of Contaminant Sorption by Soils and Sediments." The PRB media is composed of organic (alfalfa and wood chips) and inorganic (sand) components primarily designed to remove Se and metals from the mine water. These components were previously used by NewFields in a previous confidential project. These batch leach tests were also used to identify chemicals that might be released from the PRB media components including unwanted/unexpected by-products from the media/mine water interaction. Only seep water was used in the batch leach tests primarily due to the higher concentration of Se in the seep water compared to groundwater.

Briefly the test was conducted as follows: 
Journal American Society of Mining and Reclamation, 2018 Vol.7, No.3

- A total of four samples were tested:

- Alfalfa,

○ Woodchips (in duplicate),

○ Sand,

○ A "blank" (no media added) seep water sample was also used

- Samples were placed in capped $1 \mathrm{~L}$ bottles maintaining a constant aqueous volume, contaminant concentration, and media mass,

- After shaking for 24 hours, the leachate was collected by vacuum filtration and then analyzed for metals, Se and other analytes, and

- The data was analyzed to determine which constituents (metals, major cations and anions, selenium and selenium species) in the seep water increased, decreased, or remained the same while in contact with the media components.

\section{Column Leach Studies}

In comparison to the batch leach testing, the column testing was designed to yield selenium attenuation rates, provide a better opportunity to examine products of the reactions (currently proposed as part of the field pilot), and to provide useful information concerning long-term performance of the PRB mixture.

A set of multiport columns, (Fig. 1), were setup for Se removal from mine seep water and groundwater influent. The columns were constructed from 2-inch diameter, 30-inch tall, clear PVC pipe and had 5 ports: 1 influent port, 3 ports within the bed equally spaced over the column height, and 1 effluent port. The columns were packed with the media mixture of 50\% sand, $12.5 \%$ alfalfa, and $37.5 \%$ wood chips by volume. Two replicate columns were constructed to test seep water and two replicate columns were constructed to test groundwater. Water was pumped from the bottom of the column to the top in an up-flow configuration. 


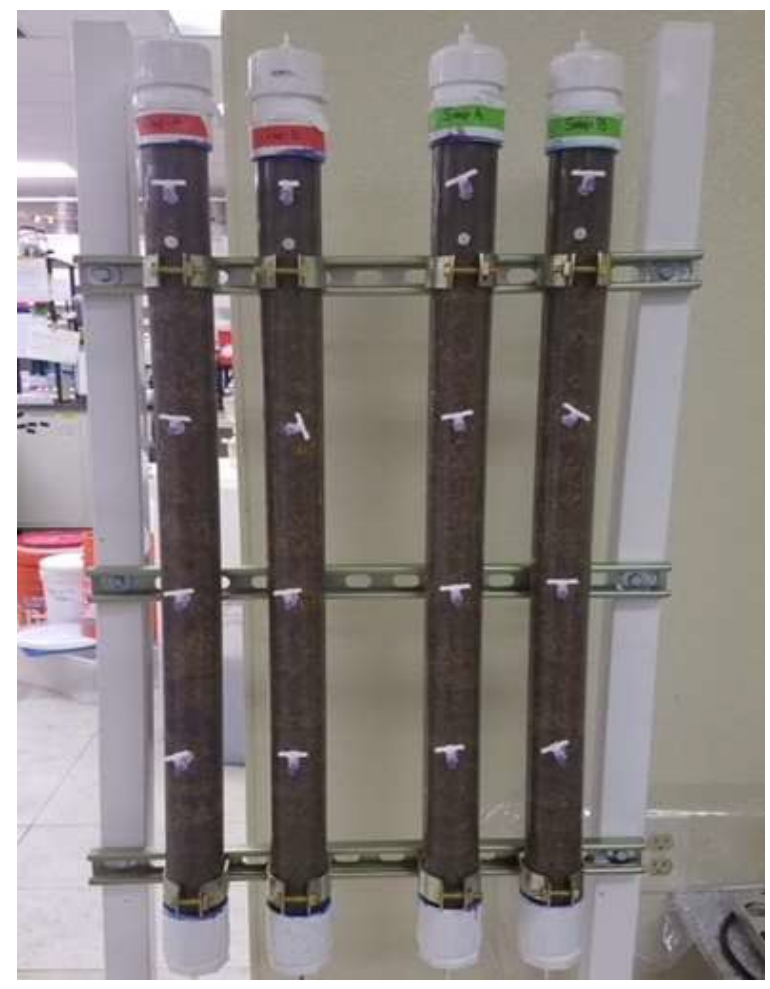

Figure 1. Multiport column setup.

The column study was designed to test a hydraulic retention time (HRT) of 12 hours initially. This was done by first filling the empty column with water to determine the total bed volume. Then the column was emptied and packed with media and weighed after obtaining a tare weight for the column. Water was added to the packed column, keeping track of volume of water added and weight. At saturation, the pore volume was computed based on the water added to the packed media column. For these columns, the bed volume was about $1540 \mathrm{ml}$ and the pore volume about $650 \mathrm{ml}$, resulting in a porosity of 0.42 . To achieve a contact time of 12 hours in the column the pump was set to a flow rate of about $0.9 \mathrm{ml} / \mathrm{min}$ resulting in a 12-hr. time to pass $650 \mathrm{ml}$ through the column (Table 1), or:

$$
0.9 \mathrm{ml} / \mathrm{min} \times 60 \mathrm{~min} / \mathrm{hr} \times 12 \mathrm{hr}=648 \mathrm{ml}=1 \text { pore volume }(\mathrm{PV})
$$

Eq 1

The columns for each influent water were replicated (Table1). The discussion of the column effluent data, however was based on the means from the two replicate columns. 
redox potential and $\mathrm{pH}$ conditions, which are also important parameters affecting the removal of inorganic species in PRBs.

Methods of Analysis

The methods of analysis for all media and samples is described below.

Solid Samples - The solid media were digested (EPA 3050B) in a strong acid and the extract was analyzed for metals using EPA methods ICP-AES (6010) or ICP-MS (6020). Mercury was analyzed using cold-vapor atomic absorption (7471). The laboratory results and attendant QA/QC information for all the samples submitted for each study can be found as an attachment to this document.

Aqueous Samples - Influent, effluent and batch leach aqueous samples were analyzed for metals and major cations using EPA methods ICP-AES (6010) or ICP-MS (6020). Selenium was speciated into total Se, Se, ${ }^{\mathrm{IV}}$ and Se ${ }^{\mathrm{VI}}$ via Method SM 3114C. Dissolved Se was determined by the same method after filtering the sample through a 0.45 micron-sized nylon filter. Since regulatory limits are typically compared to non-filtered samples, discussion of total Se in this paper refers to the non-filtered total Se species. As a performance check on total Se, occasional samples were also analyzed for total Se by EPA Method 200.8.

Other methods include: Sulfate, chloride, fluoride, phosphate, nitrate/nitrite (EPA Method 300.0), alkalinity (Method 12 SM20-2320B), and iron, manganese and aluminum (EPA Method 200.7).

Field parameters were accomplished with a YSI multiprobe for $\mathrm{pH}$, electrical conductivity (EC), total dissolved solids (TDS), and oxidation-reduction potential (ORP) using standard calibration and accuracy/precision checks.

\section{$\underline{\text { Results and Discussion }}$}

\section{Characterization of Water and PRB Media Components}

The elemental composition of the solid media was not expected to affect the quality of the water in the column or batch studies other than to provide a suitable environment for anoxic/anaerobic conditions to develop, a requirement for microbial selenium reduction. Metal concentrations in the solid media are low and the media itself is not likely a source of trace metals to the effluent other than iron and manganese which can mobilize in reducing environments. Table 2 displays the chemical composition of the media solids; sand, wood chips and alfalfa. The data in 
the table shows that the trace metal content of the materials is low. For example, Cd is less than $0.2 \mathrm{mg} / \mathrm{kg}$ in each material; $\mathrm{Cr}$ is less than $0.6 \mathrm{mg} / \mathrm{kg}$ in the alfalfa and wood chip samples and less than $5 \mathrm{mg} / \mathrm{kg}$ in the sand material. Cobalt is $1 \mathrm{mg} / \mathrm{kg}$ or less in all three samples. The $\mathrm{Cu}$ content is highest in the alfalfa material $(5.96 \mathrm{mg} / \mathrm{kg}$ ), and less than $3 \mathrm{mg} / \mathrm{kg}$ in the sand and wood chip material. Zinc ranges from 6 to $14 \mathrm{mg} / \mathrm{kg}$ in all three samples and $\mathrm{Pb}$ is less than $3 \mathrm{mg} / \mathrm{kg}$ in the three different materials. The two organic samples, alfalfa and wood chips, have Al contents of 38 and $15 \mathrm{mg} / \mathrm{kg}$, respectively, while the sand sample contains about $1200 \mathrm{mg} / \mathrm{kg} \mathrm{Al}$. The Fe contents follow the same trend as the aluminum content in which the organic materials have less than 65 $\mathrm{mg} / \mathrm{kg}$ Fe compared to $3130 \mathrm{mg} / \mathrm{kg}$ Fe in the sand sample. Arsenic is less than $2.5 \mathrm{mg} / \mathrm{kg}$ in all samples and Se is less than $4.0 \mathrm{mg} / \mathrm{kg}$ in all samples.

Prior to sampling, historic monitoring results for seep water and groundwater (not included in this report) were evaluated to understand the range of potential selenium concentrations in water requiring treatment at the mine. Seep water was selected for use in the bench study because it contains relatively high levels of selenium, usually greater than $4 \mathrm{mg} / \mathrm{L}$ and often near $10 \mathrm{mg} / \mathrm{L}$. Groundwater was selected because it represents influent with low Se levels, ranging from $1.5 \mathrm{mg} / \mathrm{L}$ to just less than $1 \mathrm{mg} / \mathrm{L}$. The analytical results for the mine seep and groundwater destined for use in the PRB bench study are shown in Table 3.

One sample of the test waters was taken prior to shipment and two sets of subsamples were collected after the water arrived at the testing lab but prior to beginning the leach tests. The "Batch Blank" sample was analyzed in conjunction with the batch leach tests. The Se data in Table 3 indicate that Se concentrations decreased during shipping and storage with the mine seep water exhibiting the largest changes. The initial Se concentration in the mine seep water was $4.84 \mathrm{mg} / \mathrm{L}$ and $1.11 \mathrm{mg} / \mathrm{L}$ in the groundwater sample. Selenium in the seep water decreased from $4.84 \mathrm{mg} / \mathrm{L}$ to $2.49 \mathrm{mg} / \mathrm{L}$ while Se in the groundwater sample decreased to about $0.8 \mathrm{mg} / \mathrm{L}$. Roughly $50 \%$ of the total Se in the mine seep water was lost during storage. The reason for the loss is not entirely clear but could be due to changing ORP (which trended to reducing conditions) in the stored water that resulted in reduction of Se to elemental Se and loss from solution. As noted earlier, the mine seep water was spiked to a higher concentration before commencement of the column studies. 
Journal American Society of Mining and Reclamation, 2018 Vol.7, No.3

Table 2. Chemical composition of PRB media components.

\begin{tabular}{|c|c|c|c|}
\hline \multicolumn{4}{|c|}{$\begin{array}{c}\text { Chemical Composition of the PRB Media Permeable } \\
\text { Bench-Scale Treatability Study } \\
\text { Conda/Woodall Mountain Mine }\end{array}$} \\
\hline Analyte & Alfalfa & Wood & Sand \\
\hline \multicolumn{4}{|c|}{ Metals (mg/kg) } \\
\hline Aluminum & $38.1 \mathrm{Jt}$ & $14.9 \mathrm{J+}$ & $1240 \mathrm{J+}$ \\
\hline Antimony & $2 U J$ & $2 U J$ & $2 U J$ \\
\hline Arsenic & $2.5 \cup J$ & $2.5 \cup J$ & $2.5 \cup J$ \\
\hline Barium & $9.96 \mathrm{~J}$ & $11.2 \mathrm{~J}$ & $34.1 \mathrm{~J}$ \\
\hline Beryllium & $0.2 \mathrm{UJ}$ & $0.2 \mathrm{UJ}$ & $0.2 \mathrm{UJ}$ \\
\hline Boron & $19.5 \mathrm{~J}$ & $4 \mathrm{UJ}$ & $4 \mathrm{UJ}$ \\
\hline Cadmium & $0.2 \mathrm{UJ}$ & $0.21 \mathrm{~J}$ & $0.2 \mathrm{~J}$ \\
\hline Chromium & $0.6 \mathrm{UJ}$ & $0.6 \mathrm{UJ}$ & $4.26 \mathrm{~J}$ \\
\hline Cobalt & 0.6 UJ & $0.6 \cup J$ & $1.01 \mathrm{~J}$ \\
\hline Copper & $5.96 \mathrm{~J}$ & $I U J$ & $2.94 \mathrm{~J}$ \\
\hline Iron & $66.4 \mathrm{~J}$ & $28.7 \mathrm{~J}$ & $3130 \mathrm{~J}$ \\
\hline Lead & $0.8 \mathrm{UJ}$ & $1.6 \mathrm{~J}$ & $2.6 \mathrm{~J}$ \\
\hline Manganese & $14.3 \mathrm{~J}$ & $34 \mathrm{~J}$ & $122 \mathrm{~J}$ \\
\hline Mercury & $0.033 \mathrm{UJ}$ & $0.033 \mathrm{UJ}$ & $0.033 \mathrm{UJ}$ \\
\hline Molybdenum & $0.8 \mathrm{UJ}$ & $0.8 \mathrm{UJ}$ & $0.8 \mathrm{UJ}$ \\
\hline Nickel & $I U J$ & $I U J$ & $2.58 \mathrm{~J}$ \\
\hline Selenium & $4 \mathrm{UJ}$ & $4 \mathrm{UJ}$ & $4 \mathrm{UJ}$ \\
\hline Silver & $0.5 \mathrm{U}$ & $0.5 \mathrm{U}$ & $0.5 \mathrm{U}$ \\
\hline Thallium & $1.5 \cup J$ & $1.5 \mathrm{UJ}$ & $1.5 \mathrm{UJ}$ \\
\hline Uranium & $0.5 \mathrm{UJ}$ & $0.5 \mathrm{UJ}$ & $0.5 \mathrm{UJ}$ \\
\hline Vanadium & $0.5 \cup J$ & $0.5 \cup J$ & $5.81 \mathrm{~J}$ \\
\hline Zinc & $14 \mathrm{~J}$ & $6.3 \mathrm{~J}$ & $10 \mathrm{~J}$ \\
\hline \multicolumn{4}{|l|}{ Notes: } \\
\hline \multicolumn{4}{|l|}{$\mathrm{mg} / \mathrm{kg}$ - milligrams per kilogram } \\
\hline \multicolumn{4}{|c|}{$\begin{array}{l}\text { J - Concentration is considered estimated. "+" indicates value may be } \\
\text { biased high. "-" indicates value may be biased low. } \\
\text { - Concentration is below the reporting limit, reporting limit shown. }\end{array}$} \\
\hline
\end{tabular}


Journal American Society of Mining and Reclamation, 2018 Vol.7, No.3

Table 3. Chemical composition of mine water: Seep and Groundwater samples Pre- and PostShipment Composition.

\begin{tabular}{|c|c|c|c|c|c|c|}
\hline \multirow{2}{*}{ Analyte } & \multicolumn{3}{|c|}{ Northeast Seep \#5 Pond Water } & \multicolumn{3}{|c|}{ Groundwater } \\
\hline & \multirow{2}{*}{$\begin{array}{c}\text { Pre-Shipment I } \\
1 \mathrm{I} / \mathrm{I} / 2017\end{array}$} & \multicolumn{2}{|c|}{ Post-Shipment } & \multirow{2}{*}{$\begin{array}{c}\text { Pre-Shipment I } \\
1 \mathrm{I} / \mathrm{I} / 2017\end{array}$} & \multicolumn{2}{|c|}{ Post-Shipment } \\
\hline Collection Date & & $12 / 14 / 2017$ & $12 / 28 / 2017$ & & $12 / 14 / 2017$ & $12 / 28 / 2017$ \\
\hline \multicolumn{7}{|c|}{ Selenium $(\mathrm{mg} / \mathrm{L})$} \\
\hline Total Selenium (by Method SM3II4C) & 4.84 & 3.37 & 2.49 & 1.11 & 0.944 & 0.808 \\
\hline Selenium IV & NA & 1.31 & 1.28 & NA & 0.043 & 0.264 \\
\hline Selenium VI & NA & $3 U$ & $1.21 \mathrm{~J}$ & NA & 0.901 & 0.544 \\
\hline Dissolved Selenium & $4.95 \mathrm{UJ}$ & 3.8 & 2.64 & $4.95 \mathrm{UJ}$ & 0.97 & 0.889 \\
\hline \multicolumn{7}{|c|}{ Dissolved Metals (mg/L) } \\
\hline Aluminum & $0.04 \mathrm{U}$ & $0.44 \mathrm{~J}-$ & \multirow{21}{*}{ NA } & $0.04 \mathrm{U}$ & $0.52 \mathrm{~J}-$ & \multirow{21}{*}{ NA } \\
\hline Antimony & $0.00119 \mathrm{~J}$ & $0.006 \mathrm{U}$ & & $0.00026 \mathrm{~J}$ & $0.006 \mathrm{U}$ & \\
\hline Arsenic & $0.0012 \mathrm{~J}$ & $0.01 \mathrm{U}$ & & $0.00086 \mathrm{~J}$ & $0.01 \mathrm{U}$ & \\
\hline Barium & $0.0243 \mathrm{UJ}$ & 0.029 & & $0.0243 \mathrm{UJ}$ & 0.048 & \\
\hline Beryllium & $0.001 \mathrm{U}$ & $0.004 \mathrm{U}$ & & $0.001 \mathrm{U}$ & $0.004 \mathrm{U}$ & \\
\hline Boron & $0.065 \mathrm{UJ}$ & $0.2 \mathrm{UJ}-$ & & $0.065 \mathrm{UJ}$ & $0.2 \mathrm{UJ}-$ & \\
\hline Cadmium & 0.00189 & $0.004 \mathrm{U}$ & & 0.00665 & 0.01 & \\
\hline Chromium & $0.0019 \mathrm{U}$ & $0.02 \mathrm{U}$ & & $0.0019 \mathrm{U}$ & $0.02 \mathrm{U}$ & \\
\hline Copper & $0.0023 \mathrm{U}$ & $0.04 \mathrm{U}$ & & $0.0035 \mathrm{~J}$ & $0.04 \mathrm{U}$ & \\
\hline Cobalt & $0.0013 \mathrm{U}$ & $0.01 \mathrm{U}$ & & $0.0024 \mathrm{~J}$ & $0.01 \mathrm{U}$ & \\
\hline Iron & $0.045 \mathrm{U}$ & $0.6 \mathrm{U}$ & & $0.045 \mathrm{U}$ & $0.6 \mathrm{U}$ & \\
\hline Lead & $0.0052 \mathrm{U}$ & $0.01 \mathrm{U}$ & & $0.0052 \mathrm{U}$ & $0.01 \mathrm{U}$ & \\
\hline Manganese & $0.046 \mathrm{UJ}$ & 0.13 & & 4.82 & 3.8 & \\
\hline Mercury & $0.000076 \mathrm{U}$ & $0.001 \mathrm{U}$ & & $0.000076 \mathrm{U}$ & $0.001 \mathrm{U}$ & \\
\hline Molybdenum & 0.067 UJ & 0.067 & & 0.067 UJ & 0.029 & \\
\hline Nickel & $0.343 \mathrm{UJ}$ & 0.400 & & $0.343 \mathrm{UJ}$ & 0.12 & \\
\hline Silver & $0.0024 \mathrm{U}$ & $0.01 \mathrm{U}$ & & $0.0024 \mathrm{U}$ & $0.01 \mathrm{U}$ & \\
\hline Thallium & $0.000039 \mathrm{~J}$ & $0.002 \mathrm{U}$ & & $0.000042 \mathrm{~J}$ & $0.002 \mathrm{U}$ & \\
\hline Uranium & $0.0213 \mathrm{UJ}$ & $0.05 \mathrm{U}$ & & $0.0213 \mathrm{UJ}$ & $0.05 \mathrm{U}$ & \\
\hline Vanadium & $0.0179 \mathrm{UJ}$ & $0.0179 \mathrm{UJ}$ & & $0.0179 \mathrm{UJ}$ & $0.02 \mathrm{U}$ & \\
\hline Zinc & $0.395 \mathrm{UJ}$ & 0.46 & & $0.395 \mathrm{UJ}$ & $0.2 \mathrm{U}$ & \\
\hline \multicolumn{7}{|c|}{ Dissolved Cations/Anions ( $\mathrm{mg} / \mathrm{L}$, or as noted) } \\
\hline Alkalinity as $\mathrm{CaCO} 3$ & 228 & 240 & \multirow{16}{*}{ NA } & 234 & 220 & \multirow{16}{*}{ NA } \\
\hline Bicarbonate & 228 & 240 & & 234 & 220 & \\
\hline Carbonate & IU & $20 U$ & & IU & $20 U$ & \\
\hline Hardness as $\mathrm{CaCO} 3$ & 2333 & NA & & 2160 & NA & \\
\hline Chloride & 11 & 20 & & 10.9 & 13 & \\
\hline Sulfate & 2220 & 2300 & & 2100 & 2200 & \\
\hline Phosphate & 32 & $5 \mathrm{U}$ & & 1.46 & $5 \mathrm{U}$ & \\
\hline Fluoride & 1.17 & $2.5 \mathrm{U}$ & & 0.528 & $2.5 \mathrm{U}$ & \\
\hline Nitrate/Nitrite as $\mathrm{N}$ & 0.123 & $2.5 \mathrm{U}$ & & 0.588 & $2.5 \mathrm{U}$ & \\
\hline Ammonia as $\mathrm{N}$ & $0.01 \mathrm{~J}$ & $0.89 \mathrm{~J}$ & & 0.046 & $0.10 \mathrm{UJ}$ & \\
\hline Calcium & 506 & 450 & & 499 & 440 & \\
\hline Magnesium & $256 \mathrm{UJ}$ & 280 & & $256 \mathrm{UJ}$ & 230 & \\
\hline Sodium & 25.9 & NA & & 22.9 & NA & \\
\hline Potassium & 5.02 & 6.9 & & 5.27 & 6.9 & \\
\hline TDS & 3230 & NA & & 3120 & NA & \\
\hline TSS & 37 & NA & & 132 & NA & \\
\hline TOC & 8.29 & 10 & 8.61 & 3.66 & 3.4 & 3.6 \\
\hline \multicolumn{7}{|c|}{ Field Parameters } \\
\hline Ferrous Iron $(\mathrm{mg} / \mathrm{L})$ & NA & $0.01 \mathrm{U}$ & NA & NA & $0.01 \mathrm{U}$ & NA \\
\hline Dissolved Oxygen (mg/L) & NA & 0.7 & 1.6 & NA & 6.6 & 5.0 \\
\hline Conductivity $(\mathrm{mS} / \mathrm{cm})$ & NA & 3.22 & NA & NA & 3.02 & $\mathrm{~N} / \mathrm{A}$ \\
\hline ORP $(m V)$ & NA & 256 & -86 & NA & 361 & 88 \\
\hline Temperature $\left({ }^{\circ} \mathrm{C}\right)$ & NA & 15 & NA & NA & 15 & NA \\
\hline $\mathrm{pH}(\mathrm{su})$ & 7.35 & 7.35 & NA & 6.88 & 6.68 & NA \\
\hline \multicolumn{7}{|c|}{$\begin{array}{l}\text { Notes: } \\
\text { PV - pore volumes; mg/L - milligrams per liter; TDS - total dissolved solids; TSS - total suspended solids; TOC - total organic carbon; mS/cm - microSiemens } \\
\text { per centimeter; mV - millivolts; OC - degrees Celsius; su - standard units; mg/L - milligrams per liter; }\end{array}$} \\
\hline $\begin{array}{l}\text { PV - pore volumes; mg/L - milligrams per liter; } \\
\text { per centimeter; mV - millivolts; OC - degrees C } \\
\text { NA - not analyzed; ORP - oxygen reducing pote } \\
\text { J - Concentration is considered estimated. "+" in } \\
\text { below the reporting limit, reporting limit shown } \\
\text { I Simplot samples from groundwater event on }\end{array}$ & $\begin{array}{l}\text { DS - total dissolve } \\
\text { sius; su - standard } \\
\text { tial } \\
\text { dicates value may } \\
\text { //1/17: SVL\# X7K }\end{array}$ & $\begin{array}{l}\text { ds; TSS - tota } \\
; \text { mg/L - millig }\end{array}$ & $\begin{array}{l}\text { spended solid } \\
\text { s per liter; } \\
\text { cates value m }\end{array}$ & ; TOC - total org & ic carbon; $\mathrm{mS}$ & roSiemens \\
\hline
\end{tabular}


Selenium VI accounted for about $90 \%$ of the total Se in the initial mine water samples. In subsequent sampling, $\mathrm{Se}^{\mathrm{IV}}$ and $\mathrm{Se}^{\mathrm{VI}}$, had nearly equivalent concentrations (i.e. $\mathrm{Se}^{\mathrm{VI}}$, decreased and $\mathrm{Se}^{\mathrm{IV}}$ increased).

Aside from the Se concentrations in the seep water, no other trace metal(oid)s appeared to be elevated and many were at the limit of detectability, $\mathrm{Zn}$ and $\mathrm{Cu}$ were less than $0.5 \mathrm{mg} / \mathrm{L}$. Groundwater dissolved metal concentrations were also very low, except for $\mathrm{Mn}$ which had a concentration of nearly $5 \mathrm{mg} / \mathrm{L}$.

The major cation and anion composition of the seep and groundwater samples were also determined. Fig. 2 and Table 3 show that the seep and groundwater samples are very similar chemically. These waters are both neutral $\mathrm{pH}$, calcium sulfate type waters with lesser amounts of sodium/magnesium bicarbonate type profiles. Sulfate concentrations in both waters exceeded 2000 $\mathrm{mg} / \mathrm{L}$ (about $2200 \mathrm{mg} / \mathrm{L}$ ) with calcium and magnesium ranging up to $500 \mathrm{mg} / \mathrm{L}$ and bicarbonate ranging up to $250 \mathrm{mg} / \mathrm{L}$. The $\mathrm{NO}_{3}, \mathrm{Fl}$, and $\mathrm{Cl}$ concentrations are all less than $50 \mathrm{mg} / \mathrm{L}$ in both samples.

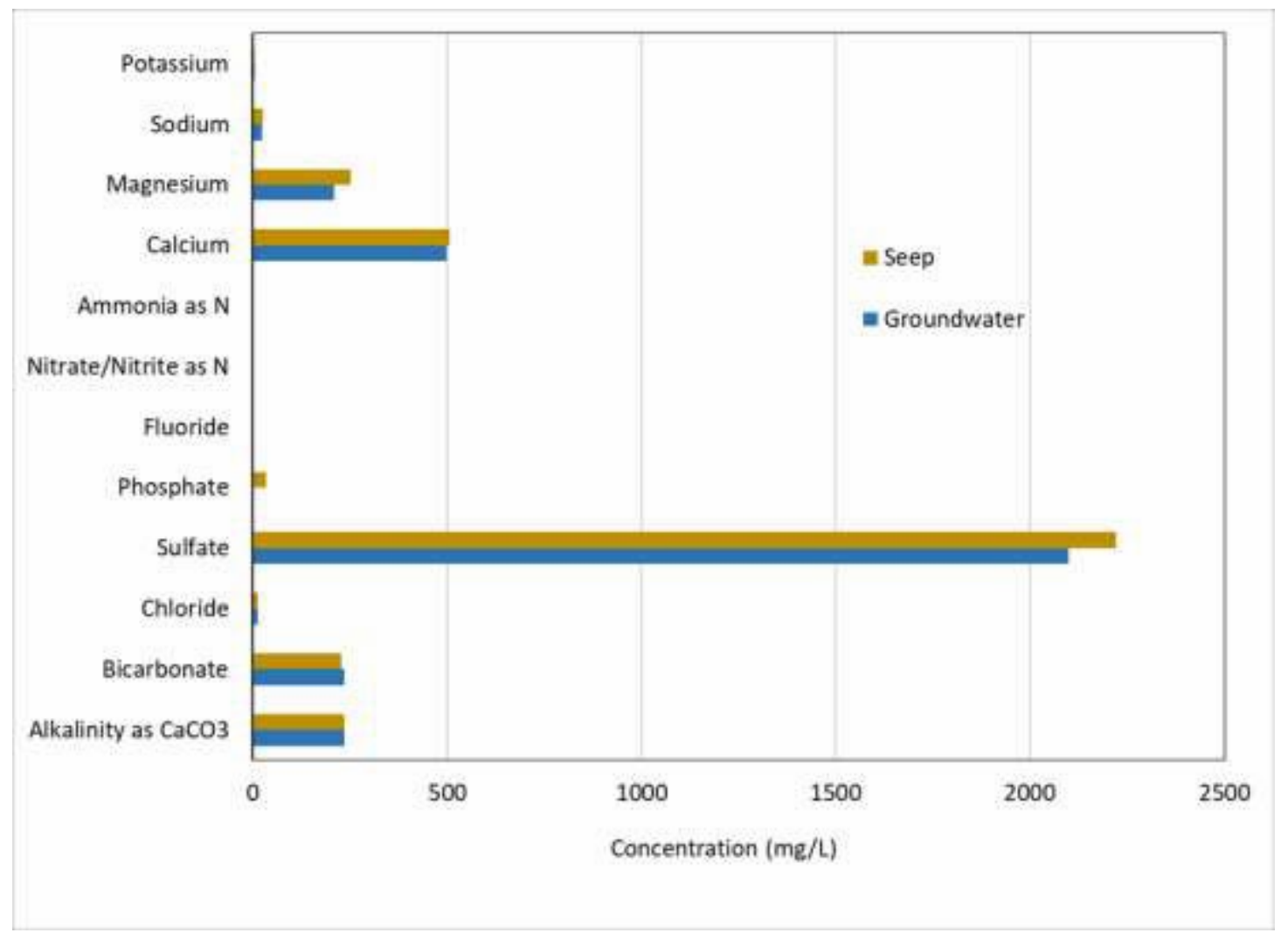

Figure 2. Major cation and anion chemistry in Seep water and Groundwater Samples 
To summarize the chemical characterization of the site water and media (sand, alfalfa, and wood chips):

- The solid media used in the batch and column studies have low trace metal contents and low As and Se concentrations (not detectable above $4 \mathrm{mg} / \mathrm{kg}$ ).

- The solid media contains $\mathrm{Fe}, \mathrm{Mn}$, and $\mathrm{Al}$ in appreciable quantities. Iron ranges from 29 $\mathrm{mg} / \mathrm{kg}$ in woody material to $3100 \mathrm{mg} / \mathrm{kg}$ in the sand, while $\mathrm{Mn}$ ranges from $14 \mathrm{mg} / \mathrm{kg}$ in the alfalfa to $122 \mathrm{mg} / \mathrm{kg}$ in the sand. Aluminum ranges up to $1200 \mathrm{mg} / \mathrm{kg}$ in the sand component. As such the media may effect changes in water quality under reducing conditions which could increase soluble Fe and Mn.

- The two mine water samples, mine seep and groundwater, are chemically very similar. They are near neutral in $\mathrm{pH}$, are dominated by $\mathrm{Ca}$, sulfate and bicarbonate alkalinity. The water samples do not contain elevated metal contents but do contain Se at appreciable levels of about $4.5 \mathrm{mg} / \mathrm{L}$ (seep water) and about $1 \mathrm{mg} / \mathrm{L}$ (groundwater).

- During the shipping and holding time, there was a decline in Se concentration in the waters, especially the mine seep water. This loss of Se prompted a decision to spike the seep water sample with additional Se to counter the loss during holding. The Se spiked sample was used in the column tests only.

\section{Batch Leach Study}

Batch leach test results are presented in Table 4 and Fig. 3. The batch testing showed that about $98 \%$ of the Se in the seep water was removed within the short time period of the study ( $24 \mathrm{hr})$ by the alfalfa component alone. Selenium concentrations were not appreciably affected by mixing seep water with either the wood chips or sand components of the media.

The initial concentration of Se in the mixtures, as determined from the seep water blank, was $3.21 \mathrm{mg} / \mathrm{L}$, while after mixing with media, Se was reduced to $0.02 \mathrm{mg} / \mathrm{L}$ in alfalfa but only $2.92 \mathrm{mg} / \mathrm{L}$ in the wood chips and sand-seep water mixtures (Table 4). This suggests that the alfalfa readily creates reducing conditions necessary for Se reduction to take place. Removal of Se (and Se species) from seep water by sand and wood chips is minimal, if at all, over the same time frame (Fig. 3). The sand is a relatively inert component and should have little effect on Se except possibly through adsorption to iron coatings that probably exist on the sand grains. Its primary use in the 
Table 4. Table of batch leach results chemical composition.

\begin{tabular}{|c|c|c|c|c|c|}
\hline \multicolumn{6}{|c|}{$\begin{array}{c}\text { Batch Leach Test Results, Collected I2/28/17 } \\
\text { Permeable Reactive Barrier Bench-Scale Treatability Study } \\
\text { Conda/Woodall Mountain Mine }\end{array}$} \\
\hline Analyte & $\begin{array}{l}\text { Seep Water } \\
\text { Blank }\end{array}$ & Alfalfa & Wood Chips & $\begin{array}{l}\text { Wood Chips } \\
\text { Dup }\end{array}$ & Sand \\
\hline \multicolumn{6}{|c|}{ Selenium and Species $(\mathrm{mg} / \mathrm{L})$} \\
\hline $\begin{array}{c}\text { Total Selenium } \\
\text { (by Method SM } 31 \mid 4 C \text { ) }\end{array}$ & 3.21 & $0.0200 \mathrm{U}$ & 2.92 & 2.88 & 2.92 \\
\hline Selenium IV & 1.12 & 0.0141 & 0.538 & 0.555 & 0.536 \\
\hline Selenium VI & $2.20 \mathrm{U}$ & 0.006 UI & 2.38 & 2.33 & 2.38 \\
\hline \multicolumn{6}{|c|}{ Dissolved Metals $(\mathrm{mg} / \mathrm{L})$} \\
\hline Aluminum & $0.08 \mathrm{U}$ & $0.16 \mathrm{U}$ & $0.08 \mathrm{U}$ & $0.08 \mathrm{U}$ & $0.08 \mathrm{U}$ \\
\hline Antimony & $0.003 \mathrm{U}$ & $0.003 \mathrm{U}$ & $0.003 \mathrm{U}$ & $0.003 \mathrm{U}$ & $0.003 \mathrm{U}$ \\
\hline Arsenic & $0.003 \mathrm{U}$ & 0.00344 & $0.003 \mathrm{U}$ & $0.003 \mathrm{U}$ & 0.0031 \\
\hline Barium & 0.0243 & 0.167 & 0.413 & 0.45 & 0.0775 \\
\hline Beryllium & $0.002 \mathrm{U}$ & $0.004 \mathrm{U}$ & $0.002 \mathrm{U}$ & $0.002 \mathrm{U}$ & $0.002 \mathrm{U}$ \\
\hline Boron & 0.065 & 0.674 & 0.061 & 0.061 & 0.066 \\
\hline Cadmium & $0.0002 \mathrm{U}$ & $0.0002 \mathrm{U}$ & 0.00077 & 0.00081 & $0.0002 \mathrm{U}$ \\
\hline Chromium & $0.006 \mathrm{U}$ & $0.012 \mathrm{U}$ & $0.006 \mathrm{U}$ & $0.006 \mathrm{U}$ & $0.006 \mathrm{U}$ \\
\hline Copper & $0.01 \mathrm{U}$ & 0.0629 & $0.01 \mathrm{U}$ & $0.01 \mathrm{U}$ & $0.01 \mathrm{U}$ \\
\hline Cobalt & $0.006 \mathrm{U}$ & $0.012 \mathrm{U}$ & $0.006 \mathrm{U}$ & $0.006 \mathrm{U}$ & $0.006 \mathrm{U}$ \\
\hline Iron & $0.1 \cup$ & 0.209 & $0.1 \cup$ & $0.1 \mathrm{U}$ & $0.1 \mathrm{U}$ \\
\hline Lead & $0.0075 \mathrm{U}$ & $0.015 \mathrm{U}$ & $0.0075 \mathrm{U}$ & $0.0075 \mathrm{U}$ & $0.0075 \mathrm{U}$ \\
\hline Manganese & 0.046 & 0.565 & 1.25 & 1.25 & 0.0824 \\
\hline Mercury & $0.0002 \mathrm{U}$ & $0.0002 \mathrm{U}$ & $0.0002 \mathrm{U}$ & $0.0002 \mathrm{U}$ & $0.0002 \mathrm{U}$ \\
\hline Molybdenum & 0.067 & $0.016 \mathrm{U}$ & $0.008 \mathrm{U}$ & $0.008 \mathrm{U}$ & 0.067 \\
\hline Nickel & 0.343 & 0.336 & 0.155 & 0.154 & 0.242 \\
\hline Silver & $0.005 \mathrm{U}$ & $0.01 \mathrm{U}$ & $0.005 \mathrm{U}$ & $0.005 \mathrm{U}$ & $0.005 \mathrm{U}$ \\
\hline Thallium & $0.001 \mathrm{U}$ & $0.001 \mathrm{U}$ & $0.001 \mathrm{U}$ & $0.001 \mathrm{U}$ & $0.001 \mathrm{U}$ \\
\hline Uranium & 0.0213 & 0.00224 & $0.001 \mathrm{U}$ & $0.001 \mathrm{U}$ & 0.021 \\
\hline Vanadium & 0.0179 & $0.01 \mathrm{U}$ & $0.005 \mathrm{U}$ & $0.005 \mathrm{U}$ & 0.0138 \\
\hline Zinc & 0.395 & 0.59 & 0.165 & 0.159 & 0.039 \\
\hline \multicolumn{6}{|c|}{ Dissolved Cations/Anions (mg/L) } \\
\hline Alkalinity & $244 \mathrm{~J}$ & $636 \mathrm{~J}$ & $119 \mathrm{~J}$ & $116 \mathrm{~J}$ & $240 \mathrm{~J}$ \\
\hline Bicarbonate & 244 & 636 & 119 & 116 & 240 \\
\hline Carbonate & $\mathrm{IU}$ & $\mathrm{IU}$ & $\mathrm{IU}$ & $\mathrm{IU}$ & $\mathrm{IU}$ \\
\hline Chloride & 11 & 87.5 & 11.5 & 11.3 & 11 \\
\hline Sulfate & 2270 & 2390 & 2270 & 2300 & 2280 \\
\hline Phosphate & 0.137 & $51.4 \mathrm{~J}$ & $0.276 \mathrm{~J}$ & $0.306 \mathrm{~J}$ & $0.085 \mathrm{~J}$ \\
\hline Fluoride & $1.34 \mathrm{~J}-$ & $223 \mathrm{~J}-$ & $1.97 \mathrm{~J}-$ & $1.98 \mathrm{~J}-$ & $1.07 \mathrm{~J}-$ \\
\hline Nitrate as $\mathrm{N}$ & $0.05 \mathrm{UJ}$ & $0.5 \mathrm{UJ}$ & $0.5 \mathrm{UJ}$ & $0.5 \mathrm{UJ}$ & $0.056 \mathrm{~J}$ \\
\hline Nitrite as $\mathrm{N}$ & $0.05 \mathrm{UJ}$ & $0.5 \mathrm{UJ}$ & $0.5 \mathrm{UJ}$ & $0.5 \mathrm{UJ}$ & $0.05 \mathrm{UJ}$ \\
\hline Magnesium & 256 & 308 & 258 & 259 & 263 \\
\hline Sodium & 27.5 & 38 & 28.1 & 28 & 28.4 \\
\hline Potassium & 4.95 & 1060 & 20.5 & 20.3 & 5.91 \\
\hline \multicolumn{6}{|c|}{ Field Parameters } \\
\hline Dissolved Oxygen (mg/L) & 7.3 & 0.2 & 0.5 & 0.4 & 8 \\
\hline Ferrous iron $(\mathrm{mg} / \mathrm{L})$ & $<0.02$ & 0.3 & 0.03 & $<0.02$ & $<0.02$ \\
\hline ORP $(\mathrm{mV})$ & 168 & -516 & 94 & 87 & 164 \\
\hline $\mathrm{pH}$ (s.u.) & 7.56 & 5.31 & 6.43 & 6.38 & 7.76 \\
\hline Temperature $\left({ }^{\circ} \mathrm{C}\right)$ & 27 & 28 & 28 & 29 & 27 \\
\hline $\begin{array}{l}\text { Notes: } \\
\mathrm{mV} \text { - millivolts; OC - degrees Celsius; } \\
\mathrm{J} \text { - Concentration is considered estima } \\
\text { UI - Estimated by difference but true }\end{array}$ & $\begin{array}{l}\text { su - standard } \\
\text { ted. "+" indicat } \\
\text { concentration i }\end{array}$ & $\begin{array}{l}\mathrm{s} ; \mathrm{mg} / \mathrm{L}-\mathrm{mi} \\
\text { alue may be } \\
\text { elow the rep }\end{array}$ & $\begin{array}{l}\text { ed high. "-" indi } \\
\text { ing limit, report }\end{array}$ & $\begin{array}{l}\text { ates value may } \\
\text { ng limit shown. }\end{array}$ & biased low. \\
\hline
\end{tabular}


PRB is to keep the material permeable and to keep the degrading organic components from plugging the aquifer or barrier when functioning at full scale. The wood chips will eventually provide a long-term source of degradable organic material to the PRB but in this short-term study had very little effect on Se chemistry.

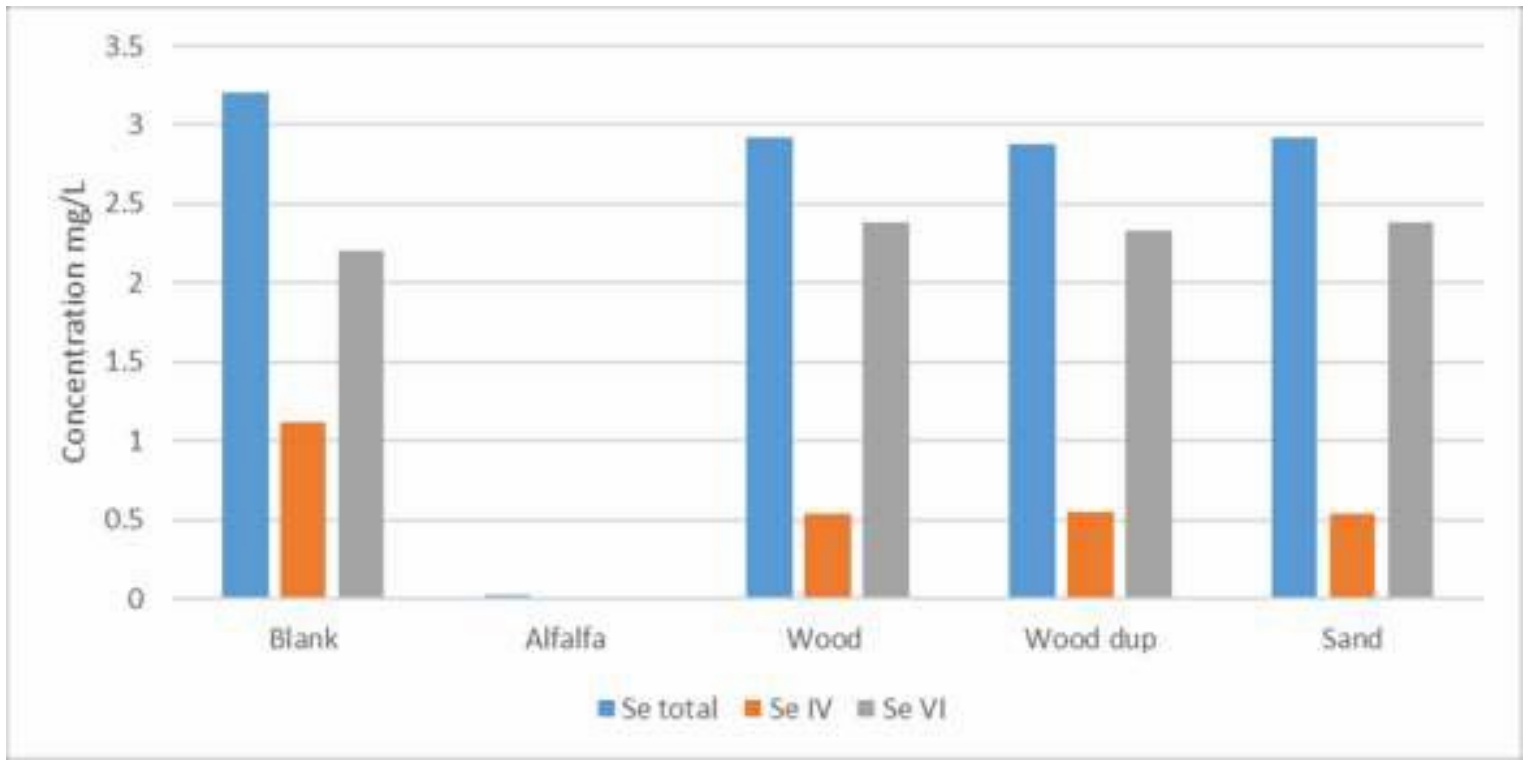

Figure 3. Effect of different media components on selenium and selenium species in batch leaching effluents.

Other changes in water chemistry were observed too. Barium $(\mathrm{Ba})$ in seep water was found to be moderately increased by contact with wood chips increasing from $0.0243 \mathrm{mg} / \mathrm{L}$ to $0.5 \mathrm{mg} / \mathrm{L}$, $\mathrm{Cu}$ in seep water was increased by contact with alfalfa from $<0.001 \mathrm{mg} / \mathrm{L}$ to $0.063 \mathrm{mg} / \mathrm{L}$, Mn was increased in seep water when in contact with all three components. The Mn content of the seep water was $0.046 \mathrm{mg} / \mathrm{L}$ and increased to $0.57 \mathrm{mg} / \mathrm{L}, 1.25 \mathrm{mg} / \mathrm{L}$ and $0.082 \mathrm{mg} / \mathrm{L}$ for alfalfa, wood chips and sand, respectively. From a water quality perspective, these are very moderate changes in solution chemistry but suggest that reducing conditions within the PRB will tend to mobilize these metals during operation.

Major cation and anion chemistry were also evaluated in batch leachate. Alkalinity in the seep water was about $250 \mathrm{mg} / \mathrm{L}$ and increased to about $650 \mathrm{mg} / \mathrm{L}$ when in contact with the alfalfa media. The increase is likely due to rapid degradation of the alfalfa and microbial metabolism resulting in production of carbon dioxide and water or, alkalinity. Alkalinity in the wood chips and sand decreased slightly due to the inherent acidity of the wood chips and sand. Phosphate was also increased when the seep water was mixed with the alfalfa. Initial phosphate concentration was $0.137 \mathrm{mg} / \mathrm{L}$ and increased to just above $51 \mathrm{mg} / \mathrm{L}$ after mixing with alfalfa. The source of the 
phosphate is likely the phosphate containing compounds (amino acids, proteins etc.) inherent in the plant vegetation that is easily mineralized during decomposition. Finally, the K concentrations also were increased significantly in the seep water-alfalfa mixture from $4.95 \mathrm{mg} / \mathrm{L}$ in the seep water to $1060 \mathrm{mg} / \mathrm{L}$ in the effluent. Plant material is rich in these macro-nutrients and most are readily mineralized or released during plant decomposition, $\mathrm{P}$ concentrations were also slightly increased in the other mixtures as well, but only by 10 to $15 \mathrm{mg} / \mathrm{L}$.

The observed chemistry and chemical changes in the batch leachates were compared to Idaho Groundwater Quality Standards (Idaho 58.01.11). Only Mn exceeded the secondary water quality standard of $0.05 \mathrm{mg} / \mathrm{L}$. No other trace metals were above the primary ground water standards.

Overall, the bench testing revealed that the primary organic substrate, alfalfa, will decompose quickly and provide a suitable environment for Se reduction to occur. The Se in the alfalfa-seep water mixture was almost completely removed in the 24-hour duration of the test. The other components, wood chips and sand, had little effect on either Se reduction or on generation of unwanted chemical constituents. The chemical changes that did occur were due to well-known structural compounds of the wood and sand media, none of which caused leaching of constituents at concentrations that exceeded Idaho water quality standards in the batch tests. The role of the wood chips and sand will be important in maintaining a longer-term source of utilizable carbon for maintaining reducing conditions and for maintaining a permeable media for water flow through the media.

\section{Column Leach Studies}

The 25 PV Results- Samples were collected from the influent and effluent at each port after 25 pore volumes had passed through the columns. The initial conditions of the influent water were similar to those described in the batch study, with the notable exception that the seep water was spiked with sodium selenate to raise the total Se concentration to about $10 \mathrm{mg} / \mathrm{L}$. This was done to better represent the historic water quality observed in the seep and because the seep water Se was decreasing during the holding time due to internal geochemical conditions allowing Se reduction to $\mathrm{Se}^{\mathrm{o}}$ to occur.

Since the columns were replicated, the discussion of the data refers to the means of the two columns at each event and at each port. Overall, the relative percent difference (RPD) for the duplicate columns was less than 13\%. (The complete set of data for both columns is available electronically). In addition, most of the discussion centers on a comparison of influent data to 
effluent data. The data for individual ports is only discussed for Se, Fe, and Mn in the 25 PV Seep Water column. There was very little difference in observed water chemistry between the different sample port collection positions along the columns allowing a simple comparison of the column efficacy for Se removal based on the column effluent sample compared only to the influent (complete port sampling data is available electronically). This indicates that once mine water entered the column, it was altered quickly by the PRB such that all water within the column was similar to the effluent chemistry. The trends in Fe and Mn are somewhat more complex.

Table 5 presents data from the 25 PV mine seep and groundwater column study. Selenium in the influent seep water sample (after spiking) was $10.04 \mathrm{mg} / \mathrm{L}$. The effluent Se from the same column was only $0.186 \mathrm{mg} / \mathrm{L}$ or a $98 \%$ Se removal by the PRB. For the groundwater column, initial Se was $1.09 \mathrm{mg} / \mathrm{L}$ while the effluent contained only about $0.02 \mathrm{mg} / \mathrm{L}$ or a $98 \%$ removal as well.

For the effluent metals determined in the column study, Al and Sb remained at the limit of detection, while Cd decreased between influent and effluent (from $0.5 \mathrm{ug} / \mathrm{L}$ to $0.4 \mathrm{ug} / \mathrm{L}$ in seep column and $4 \mathrm{ug} / \mathrm{L}$ to $<0.2 \mathrm{ug} / \mathrm{L}$ in the groundwater column), and As slightly increased between the influent and effluent ports (increased from $3.8 \mathrm{ug} / \mathrm{L}$ to $11 \mathrm{ug} / \mathrm{L}$ in the seep column and increased from $<3.8 \mathrm{ug} / \mathrm{L}$ to $24.9 \mathrm{ug} / \mathrm{L}$ in the groundwater column).

Major cation and anion chemistry were unremarkable between influent and effluent samples with the exception of alkalinity, which increased in both column types. Alkalinity in the mine seep water increased by about $100 \mathrm{mg} / \mathrm{L}$ and increased by $165 \mathrm{mg} / \mathrm{L}$ in the groundwater column. The alkalinity increase is presumably due to microbial carbon metabolism.

Table 6 displays the individual column port data for Se, Fe, and $\mathrm{Mn}$ in the mine seep water only. There were discernable changes along the column as expected due to the changing redox conditions from the influent port to the effluent port (Fig. 4 and 5). Groundwater patterns for Se, $\mathrm{Fe}$, and $\mathrm{Mn}$ are almost identical to the seep water patterns and are not shown.

In general, the port closest to the influent end of the column was slightly higher in Se compared to ports located further along the flow path in the column, reflecting the increased contact time between incoming water and the microbially-mediated reactions (Table 6). For Fe and Mn, both increased from the influent side to the effluent end of the column presumably due to the stronger reducing conditions within the column and the release of Fe and Mn from the PRB wood and sand components. 
Journal American Society of Mining and Reclamation, 2018 Vol.7, No.3

Table 5. Results of the 25 PV Column Study for Seep and Groundwater samples.

\begin{tabular}{|c|c|c|c|c|}
\hline Analyte & $\begin{array}{l}\text { Seep Water } \\
\text { Influent }\end{array}$ & $\begin{array}{l}\text { Seep Water } \\
\text { Effluent }\end{array}$ & $\begin{array}{c}\text { Ground- water } \\
\text { Influent }\end{array}$ & $\begin{array}{l}\text { Ground- } \\
\text { water } \\
\text { Influent }\end{array}$ \\
\hline \multicolumn{5}{|c|}{ Selenium Species (mg/L) } \\
\hline Total Se & 10.04 & 0.186 & 1.09 & 0.0181 \\
\hline Se IV & 2.62 & 0.0179 & 0.072 & 0.011 \\
\hline SeVI & 7.42 & 0.165 & 1.01 & 0.0072 \\
\hline \multicolumn{5}{|l|}{ Dissolved Metals (mg/L) } \\
\hline Aluminum & $<0.08$ & $<0.1$ & $<0.08$ & $<0.08$ \\
\hline Antimony & $<0.003$ & $<0.003$ & $<0.003$ & $<0.003$ \\
\hline Arsenic & $<0.00375$ & 0.0111 & $<0.00375$ & 0.0249 \\
\hline Cadmium & 0.00054 & 0.00042 & 0.00415 & $<0.0002$ \\
\hline Calcium & 533 & 540 & 535 & 545 \\
\hline Iron & $<0.1$ & 0.566 & $<0.1$ & 1.63 \\
\hline Magnesium & 258 & 265 & 221 & 224 \\
\hline Manganese & 0.0496 & 0.653 & 1.04 & 2.84 \\
\hline Potassium & 5.72 & 5.21 & 5.76 & 4.83 \\
\hline Sodium & 32.4 & 32.3 & 25.7 & 25.5 \\
\hline \multicolumn{5}{|l|}{ Dissolved Anions (mg/L) } \\
\hline Alkalinity & 255 & 349 & 217 & 382 \\
\hline Bicarb & 255 & 349 & 217 & 382 \\
\hline Carbonate & $<1$ & $<1$ & $<1$ & $<1$ \\
\hline Chloride & 11.7 & 11.4 & 11.5 & 11.2 \\
\hline Fluoride & 2.24 & 1.31 & 0.817 & 0.565 \\
\hline Sulfate & 2390 & 2435 & 2140 & 1940 \\
\hline Phosphate & 0.15 & $<0.01$ & 0.159 & 0.088 \\
\hline \multicolumn{5}{|l|}{ Field Parameters } \\
\hline Conductivity (mS/cm) & 3.28 & 3.2 & 3.04 & 2.99 \\
\hline $\mathrm{DO}(\mathrm{mg} / \mathrm{L})$ & 4.5 & 0.5 & 0.5 & 0.5 \\
\hline ORP (mv) & 170 & -180 & -215 & -173 \\
\hline pH (standard units) & 7.91 & 6.67 & 6.77 & 6.55 \\
\hline
\end{tabular}


Table 6. Results of the 25 PV seep water column for Se, Fe and Mn as measured at individual ports.

\begin{tabular}{|l|c|c|c|c|c|}
\hline Analyte & $\begin{array}{c}\text { Seep } \\
\text { Water } \\
\text { Column } \\
\text { Influent }\end{array}$ & $\begin{array}{c}\text { Seep } \\
\text { Water } \\
\text { Column } \\
\text { Port 1 }\end{array}$ & $\begin{array}{c}\text { Seep } \\
\text { Water } \\
\text { Column } \\
\text { Port 2 }\end{array}$ & $\begin{array}{c}\text { Seep } \\
\text { Water } \\
\text { Column } \\
\text { Port 3 }\end{array}$ & $\begin{array}{c}\text { Seep } \\
\text { Water } \\
\text { Column } \\
\text { Effluent }\end{array}$ \\
\hline Total Se & 10.04 & 0.275 & 0.135 & 0.197 & 0.186 \\
\hline Iron & $<0.1$ & 0.188 & 0.267 & 0.427 & 0.566 \\
\hline Manganese & 0.0496 & 0.091 & 0.179 & 0.394 & 0.653 \\
\hline
\end{tabular}

Figure 4 shows the change in influent seep water Se as it moves through the column. The initial Se was approximately $10 \mathrm{mg} / \mathrm{L}$ and decreased to $0.275 \mathrm{mg} / \mathrm{L}$ at Port 1 , to $0.135 \mathrm{mg} / \mathrm{L}$ at Port and then slightly increased to $0.197 \mathrm{mg} / \mathrm{L}$ at Port 3, and $0.186 \mathrm{mg} / \mathrm{L}$ at the effluent side.

Figure 4 also shows the changes in Fe and Mn in the seep water column. Influent Fe and Mn concentrations were $<0.1 \mathrm{mg} / \mathrm{L}$ and $0.05 \mathrm{mg} / \mathrm{L}$, respectively then increased to about $0.2 \mathrm{mg} / \mathrm{L}$ and $0.1 \mathrm{mg} / \mathrm{L}$ in Port 2 and increased continually to the effluent port concentrations of $0.57 \mathrm{mg} / \mathrm{L}$ for $\mathrm{Fe}$ and to $0.65 \mathrm{mg} / \mathrm{L}$ for $\mathrm{Mn}$. The increase in Fe and $\mathrm{Mn}$ is due to the reducing conditions in the column. The influent water is slightly oxidizing and as it enters the column, dissolved oxygen is rapidly depleted allowing $\mathrm{Fe}$ and $\mathrm{Mn}$ in the media to solubilize due to change in oxidation status of the two metals where $\mathrm{Fe}^{+3}$ reduces to $\mathrm{Fe}^{+2}$ and $\mathrm{Mn}^{+4}$ reduces to $\mathrm{Mn}^{+2}$ which are more soluble species.

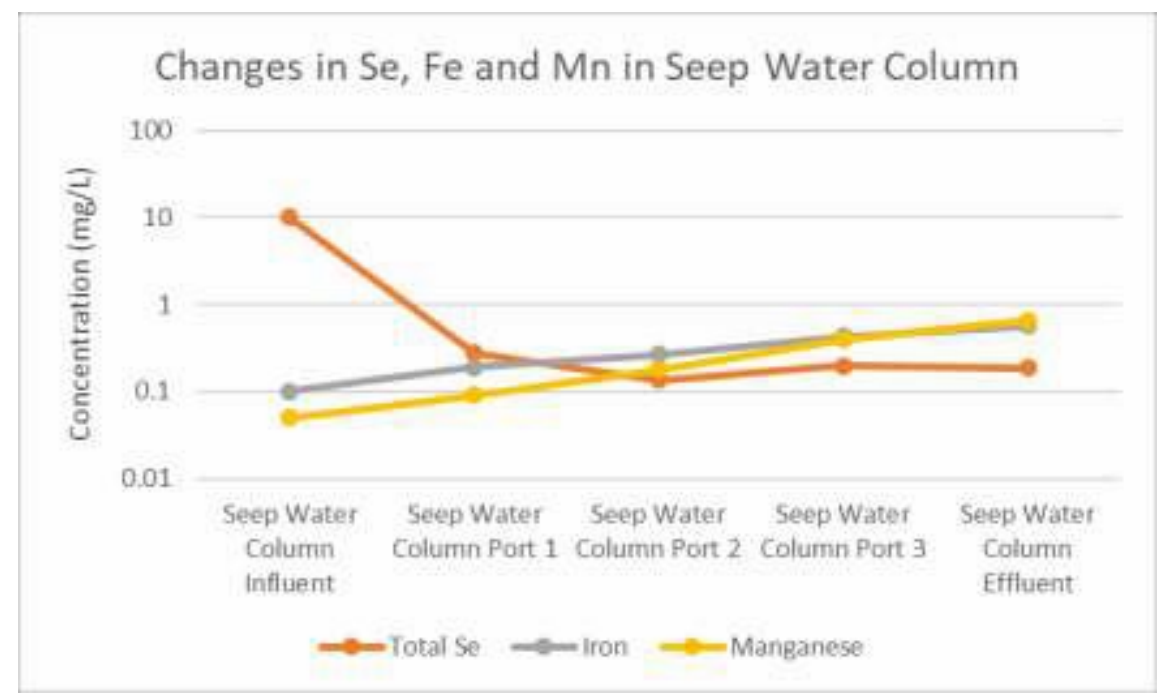

Figure 4. Changes in Se, Fe and Mn concentration from influent to effluent in Seep columns at 25 PV. 
The data from the $25 \mathrm{PV}$ collection indicates that greater than $98 \%$ Se removal has occurred within the columns. Se speciation was different for the seep water compared to groundwater. Selenate was about 10 times greater in the seep water while in the groundwater selenite was slightly greater or nearly equal to the selenate concentrations.

Overall after $25 \mathrm{PV}$, the reactive barrier, as simulated by the columns, performed consistent with the results of the batch study and with previous investigations involving PRBs for Se removal. Ninety eight percent of the initial selenium was attenuated within the column. Other observations and a summary of the $25 \mathrm{PV}$ sample results include:

- Alkalinity in both effluents (Seep and Groundwater) increased by at least $100 \mathrm{mg} / \mathrm{L}$ compared to the influent water. The increase is due to microbial carbon metabolism that converts complex carbon compounds to carbon dioxide and water which produces bicarbonate.

- Iron and Mn in the effluents of both water types increased with increasing depth in the column probably due to decreases in ORP. The increase is presumably due to reduction of $\mathrm{Fe}^{+2}$ in iron oxyhydroxides which allows dissolution of the mineral and release of $\mathrm{Fe}^{+2}$.

- Most analyte concentrations (see Se below) in the column leachate and along the column length were below Idaho water quality standards for primary contaminants, but slightly above the secondary standards for $\mathrm{Mn}$ and Fe in the effluent samples. This was expected given the reducing conditions imposed by the column conditions and the $\mathrm{Fe}$ and $\mathrm{Mn}$ contents noted in the material characterization and the batch leach studies. Overall, the different studies are consistent with respect to leachate chemistry and solubility of metals at neutral $\mathrm{pH}$.

- The 25 PV data also suggest that the 12-hour contact time (HRT) is sufficient to reduce the Se below the water quality standard of $0.05 \mathrm{mg} / \mathrm{L}$ for groundwater and almost to the water quality goal for Se in the seep water. Since the total Se in the groundwater is only $1 / 10^{\text {th }}$ that of the seep water, less contact time would be expected. The effect of additional flushing (50 PV) and a longer HRT for seep water Se are discussed in the next section. 
The 50 PV Results - At the end of the 50-pore volume (PV) interval, samples from the influent, effluent, and each port along the column were again collected and analyzed for the same constituents collected at the 25PV interval. Results are shown in Table 7. The results of the $50 \mathrm{PV}$ sampling interval are very similar to the $25 \mathrm{PV}$ results but there are some differences.

Table 7. Results of the 50 PV column study for Seep and Groundwater samples.

\begin{tabular}{|c|c|c|c|c|}
\hline Analyte & $\begin{array}{c}\text { Seep } \\
\text { Water } \\
\text { Influent }\end{array}$ & $\begin{array}{c}\text { Seep } \\
\text { Water } \\
\text { Effluent }\end{array}$ & $\begin{array}{l}\text { Ground- } \\
\text { water } \\
\text { Influent }\end{array}$ & $\begin{array}{l}\text { Ground- } \\
\text { water } \\
\text { Effluent }\end{array}$ \\
\hline \multicolumn{5}{|c|}{ Selenium Species (mg/L) } \\
\hline Total Se & 8.81 & 0.096 & 0.758 & 0.0136 \\
\hline Se IV & 2.79 & 0.039 & 0.075 & 0.0057 \\
\hline SeVI & 6.02 & 0.057 & 0.683 & 0.0085 \\
\hline \multicolumn{5}{|c|}{ Dissolved Metals (mg/L) } \\
\hline Aluminum & $<0.08$ & $<0.1$ & $<0.08$ & $<0.08$ \\
\hline Antimony & $<0.005$ & $<0.003$ & $<0.003$ & $<0.003$ \\
\hline Arsenic & $<0.0075$ & 0.0111 & $<0.00375$ & 0.0249 \\
\hline Cadmium & 0.0005 & 0.00042 & 0.00415 & $<0.0002$ \\
\hline Calcium & 539 & 571 & 537 & 561 \\
\hline Iron & $<0.1$ & $<0.1$ & $<0.1$ & $<0.1$ \\
\hline Magnesium & 276 & 269 & 227 & 228 \\
\hline Manganese & 0.054 & 0.256 & $<0.008$ & 0.324 \\
\hline Potassium & 5.44 & 5.64 & 5.4 & 5.59 \\
\hline Sodium & 33.8 & 34.5 & 26.3 & 26.8 \\
\hline \multicolumn{5}{|c|}{ Dissolved Anions (mg/L) } \\
\hline Alkalinity & 259 & 311 & 218 & 320 \\
\hline Bicarb & 259 & 311 & 218 & 320 \\
\hline Carbonate & $<1$ & $<1$ & $<1$ & $<1$ \\
\hline Chloride & 11.7 & 11.4 & 11.5 & 11.2 \\
\hline Fluoride & 1.99 & 1.72 & 0.929 & 0.85 \\
\hline Sulfate & 2500 & 2280 & 2190 & 1960 \\
\hline Phosphate & 0.146 & 0.24 & 0.158 & $<0.05$ \\
\hline \multicolumn{5}{|l|}{ Field Parameters } \\
\hline Conductivity (mS/cm) & 3.28 & 3.28 & 3.04 & 2.98 \\
\hline $\mathrm{DO}(\mathrm{mg} / \mathrm{L})$ & 4.5 & 0.5 & 4.3 & 0.5 \\
\hline ORP (mv) & 170 & -252 & 232 & -263 \\
\hline pH (standard units) & 7.91 & 7.1 & 6.88 & 6.8 \\
\hline
\end{tabular}

At $50 \mathrm{PV}$, total Se in the seep water columns decreased from $8.81 \mathrm{mg} / \mathrm{L}$ in the influent to 0.096 $\mathrm{mg} / \mathrm{L}$ in the effluent but was slightly above the water quality limit of $0.050 \mathrm{mg} / \mathrm{L}$. The seep effluent Se at $50 \mathrm{PV}$ was however, lower than that observed at $25 \mathrm{PV}(0.186 \mathrm{mg} / \mathrm{L}$ at $25 \mathrm{PV}$ compared to $0.096 \mathrm{mg} / \mathrm{L}$ at $50 \mathrm{PV})$. Groundwater effluent from the columns, on the other hand, was consistently 
lower than the $0.050 \mathrm{mg} / \mathrm{L}$ water quality limit, at 25 and $50 \mathrm{PV}$ sampling times. Selenium in the $50 \mathrm{PV}$ groundwater effluent decreased to $0.0136 \mathrm{mg} / \mathrm{L}$ compared to $0.8 \mathrm{mg} / \mathrm{L}$ Se in the influent water. Overall the HRT of 12 hours resulted in effluent Se concentrations in both columns very close or under the water quality standard of $0.050 \mathrm{mg} / \mathrm{L}$.

The metal content of the effluents from both the seep and groundwater columns was generally unremarkable. For example, $\mathrm{Al}, \mathrm{Cd}, \mathrm{Sb}$, and Fe were all close to or less than the method detection limits. Arsenic increased to $11 \mathrm{ug} / \mathrm{L}$ in the seep effluent and $24 \mathrm{ug} / \mathrm{L}$ in the groundwater effluent, very similar to the $25 \mathrm{PV}$ results. The major cation and anion chemistry were marginally affected and in general resembled that described in Table 5 for the chemistry of the 25 PV results. Alkalinity again increased in both columns by about $50 \mathrm{mg} / \mathrm{L}$ and $100 \mathrm{mg} / \mathrm{L}$ in the seep effluent and groundwater effluents, respectively. Sulfate in both effluents decreased by about $200 \mathrm{mg} / \mathrm{L}$ suggesting that some sulfate reduction could be occurring which would also explain the alkalinity increases due to microbial carbon metabolism under anaerobic conditions.

Selenium concentrations along the seep column length trended similarly to those observed in the 25 PV data but different in terms of concentration (Fig. 5). The Se concentrations at 50 PV by Port were less variable than those observed at $25 \mathrm{PV}$. Total Se in Port 1 was only $0.13 \mathrm{mg} / \mathrm{L}$ and only decreased to $0.1 \mathrm{mg} / \mathrm{L}$ in the effluent port. This may be due to the PRB column establishing a steady state or uniformly reducing conditions over the length of the column. Regardless of the process, Se in the influent is rapidly reduced within the PRB. Similar results were noted for the groundwater column.

The Fe and Mn trends in the column ports were also similar to the $25 \mathrm{PV}$ results in that there was usually a slight increase in Fe and Mn concentrations from Port 1 to the effluent Port, but the concentration increases were smaller than those observed at the $25 \mathrm{PV}$ sampling for both waters, similar to the Se trend noted above. The Mn concentration in the seep water effluent was only $1 / 3$ the concentration observed at $25 \mathrm{PV}$ and the Fe concentration in the seep water ports was only about $1 / 5^{\text {th }}$ that observed at $25 \mathrm{PV}$ in the seep water. Similar results were noted for groundwater concentrations. The lower Fe and Mn concentrations at $50 \mathrm{PV}$ suggest that soluble forms of Fe and Mn are simply being flushed from the column media resulting in better water quality with time and continued flushing. 


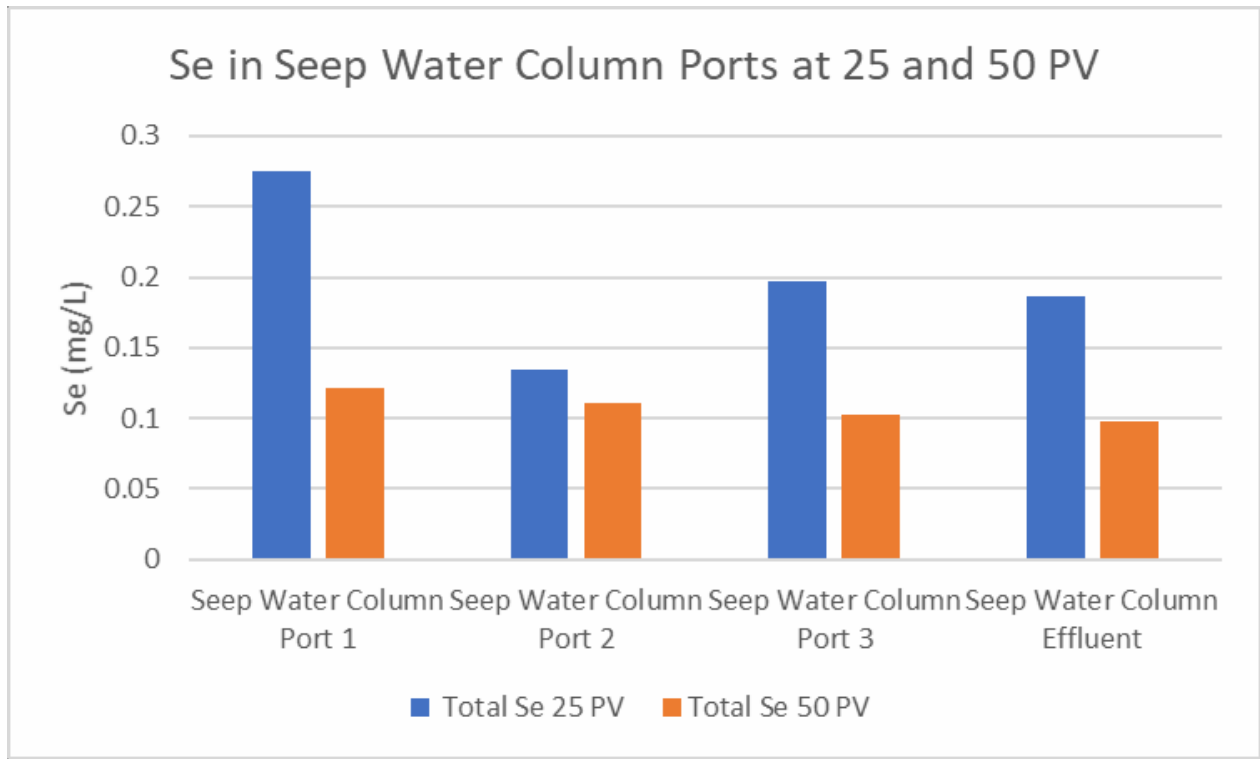

Figure 5. Comparison of 25 PV and 50 PV Se Changes in Seep Water Column

Increase Residence Times - The column study was conducted with a $12 \mathrm{hr}$ HRT via a flow rate of $0.09 \mathrm{~mL} / \mathrm{minute}$. The HRT represented the time a single PV moved from influent port to effluent port, hence the ports along the column represent residence times less than 12 hours. Based on the seep effluent data at an HRT of 12 hours, Se concentrations were still slightly above the $0.050 \mathrm{mg} / \mathrm{L}$ water quality target. Therefore, we attempted to collect an additional data point at a longer residence time ( 24 hours). To do this, the flow rate of the seep columns was reduced by $50 \%$ thereby doubling the HRT. After adjusting the flow rate to achieve the desired HRT, the seep effluent port was sampled again after 7 days (equal 7 additional PV) at the new HRT. The results are presented in Table 8 .

Table 8. Results of Se in Seep water column effluent with additional pore volumes and a 24 - hr HRT.

\begin{tabular}{|c|c|c|}
\hline \multicolumn{2}{|c|}{ Analyte Influent } & $\begin{array}{c}\text { Seep } \\
\text { Effluent }\end{array}$ \\
\hline \multicolumn{3}{|c|}{ Selenium and Species (mg/L) } \\
\hline Total Selenium & 8.81 & 0.068 \\
\hline Selenium IV & 2.79 & 0.0069 \\
\hline Selenium VI & 6.02 & 0.0601 \\
\hline
\end{tabular}

The data indicates that $\mathrm{Se}$ in the seep effluent could be reduced to $0.068 \mathrm{mg} / \mathrm{L}$ at the longer HRT, a good improvement from the $0.096 \mathrm{mg} / \mathrm{L}$ average at $50 \mathrm{PV}$ under the 12-hour HRT. It is therefore possible that further increases in HRT could result in total effluent Se decreasing below the $0.050 \mathrm{mg} / \mathrm{L}$ water quality target. 
JR Simplot intends to employ a two-stage treatment system during the field-scale treatability study that ensure sufficient residence time is achieved. The water that enters the PRB in the pilotscale system will be a combination of seep water and groundwater and therefore will have an influent selenium concentration between 1 and $10 \mathrm{mg} / \mathrm{L}$. With a mixture of groundwater and seep water, the influent concentration will be lower than the tested seep water and an HRT of $<12$ hours may be sufficient to achieve the water quality standard (or better) in the PRB effluent. In addition, a two-stage treatment system consisting of a pre-treatment PRB and then a second PRB, will ensure sufficient contact time with the PRB media to bring the influent Se concentration down to a concentration below the Idaho groundwater quality standard.

\section{$\underline{\text { Summary and Conclusions }}$}

A bench study was designed to determine the efficacy of a PRB for removing elevated Se in groundwater and mine seep water at the JR Simplot Conda/Woodall Mine in southern Idaho. The bench testing was considered a first step in the pre-design considerations for developing an engineering and geochemical strategy for long-term water treatment options at the site.

The study consisted of three main parts: (1) characterization work designed to determine the basic chemistry of the site-water under consideration for treatment and the components of the proposed PRB. This data is a requirement for establishing the initial chemical conditions for assessing the efficacy of the PRB and its components. (2) batch leaching studies designed to assess the chemistry changes that each media component is expected to contribute to the overall water chemistry of the seep or groundwater in contact with the media. This batch study also allows a determination, prior to more extensive column testing, of whether unwanted chemicals or chemical changes will occur due to contact with the PRB media. (3) column studies consisting of vertical, 2-inch columns filled with the PRB media. Seep water and groundwater were delivered to the columns in an up-flow manner under a specified flow rate to allow sufficient HRT (12 and $24 \mathrm{hr}$ ) for Se reduction to occur.

The results of the studies indicated that seep or groundwater in contact with individual media components (sand, alfalfa, wood chips) did not result in any chemical changes of concern other than those considered important for Se removal from solution. Manganese was elevated above seep water concentrations in the alfalfa and wood chip leachate but was still an order of magnitude below background groundwater levels. The alfalfa-seep water mixture resulted in very rapid removal of over $98 \%$ of the Se in the seep water due to the strong reducing conditions/carbon 
utilization produced from the alfalfa decomposition. Wood chips had very little effect on water chemistry other than slight reductions in alkalinity and $\mathrm{pH}$. The sand-seep water mixture also resulted in slight increase in arsenic probably from reduction of arsenic bearing iron minerals on sand grain surfaces via reductive dissolution. None of the observed trace metal(oid) concentrations were expected to be problematic for water quality effluent from the PRB.

The column testing results indicated Se was effectively removed from the influent solutions. The Se concentration in seep water was initially about $10 \mathrm{mg} / \mathrm{L}$ and was reduced to about $0.2 \mathrm{mg} / \mathrm{L}$ after $25 \mathrm{PV}$ and $0.1 \mathrm{mg} / \mathrm{L}$ after $50 \mathrm{PV}$. Increasing the HRT to $24 \mathrm{hr}$ (a factor of 2) decreased the Se to just above $(0.07 \mathrm{mg} / \mathrm{L})$ the $0.05 \mathrm{mg} / \mathrm{L}$ water quality goal. The groundwater Se, initially about $1 \mathrm{mg} / \mathrm{L}$, was reduced to less than 0.02 in the first 3 hours of column contact time, well below the

$0.05 \mathrm{mg} / \mathrm{L}$ water quality goal. The column testing results were also consistent with the batch leach results and the material characterization work. Solubility of primary contaminants, mainly metals, remained below the water quality goals for groundwater with only expected increases in Fe and Mn slightly above secondary water quality standards.

The HRT of 24 hours was sufficient contact time to reduce the high Se concentrations in the spiked seep water just above the Idaho water quality standard. If the PRB system treats a mixture of seep water and groundwater, resulting in a much lower influent Se concentration, then a 12 hour to 24-hour contact time or less should be sufficient to meet water quality goals.

Overall, the results indicated that the PRB composition used in the study will be an effective means of meeting water quality goals at the site. The study provided much needed informationon water and media composition information and the residence times required to accomplish Se reduction. Aside from Se reduction, no significant deleterious changes to water quality were observed when compared to primary water quality standards.

\section{Literature Cited}

ASTM Method D-4646-02. 24-h Batch-Type Measurement of Contaminant Sorption by Soils and Sediments.

Buck, B.W and J.L Jones, 2003. Interagency/Industry Coordination to Respond to Selenium Contamination at Phosphate Mines in Southeastern Idaho. Caribou-Targee National Forest. Soda Springs, ID.

Blowes, D.W., C.J. Ptacek, S.G Benner, C.W.T McRae, T.A Bennett, and R.W. Puhls. 2000. Treatment of inorganic contaminants using permeable reactive barriers. J. of Contam. Hydrol. 45(200) 123-137. https://doi.org/10.1016/S0169-7722(00)00122-4

Desborough, G., E. DeWitt, A. Meier, and G. Meeker.1999. Preliminary mineralogical and chemical studies related to potential mobility of selenium and associated elements in Phosphoria formation strata. Southeastern Idaho. USGS Open File Report 99-120. 
Herring, J.R., G. Desborough, R.G. Tysdal, and R.I. Grauch. 1999. Selenium in weathered and unweathered parts of the Meade Peak phosphatic member of the Phosphoria formation, Southeastern Idaho. Geological Society of America, Abstracts with Programs. Rocky Mountain Section. April 1999.

USEPA, 1998. Permeable Reactive Barrier Technologies for Contaminant Remediation. September. EPA, 2014. Superfund Analytical Services / Contract Laboratory Program (CLP); National Functional Guidelines.

http://www.epa.gov/superfund/programs/clp/guidance.htm (Web document). United States Environmental Protection Agency. Retrieved from http://www.epa.gov/superfund/programs/clp/guidance.htm

Formation Environmental, 2018. Email with excel spreadsheet containing Seep Water results (SDG X7K0079 from Silver Valley Laboratories). January 18, 2018.

Xia, S., S Shen, J., Lliang, and X. Xu. 2013. Study on removing selenate from groundwater by autohydrogenotrophic microorganisms. Env. Sci. Eng. 7(4) 552-558.

https://doi.org/10.1007/s11783-013-0531-2 